\title{
Numerical Study of Surfactant Effects on the Buoyancy-driven Motion of a Drop in a Tube
}

\author{
Yuanyuan Cui, Nivedita R. Gupta ${ }^{1}$ \\ Department of Chemical Engineering \\ University of New Hampshire \\ Durham, New Hampshire 03824
}

\begin{abstract}
We present results of our numerical study of the effect of surfactants on buoyancy-driven motion of drops in a tube at intermediate Reynolds numbers. The drop and bulk phases are treated as incompressible Newtonian fluids and simulated using a front-tracking method. The steady shapes and velocity-volume curves for drops ranging in drop size from $0.2-1.3$ of tube radius are determined numerically. At small Bond numbers, the velocity-volume curve shows a maximum before the velocity plateaus for large drops. As the Bond number increases, the maximum in the velocity-volume curve disappears with elongated, more streamlined drop shapes consistent with previous experimental studies. For increasing Weber numbers, the rear of the drop shows a flattening followed by the development of a negative curvature. Surfactants are modeled using a Langmuir equation of state in the adsorption-desorption limit and the effect of surfactant mass transfer, fractional coverage of surfactants, and the interfacial Peclet number on the velocity-volume curve is determined. Marangoni stress generated due to the non-uniform distribution of surfactants at the interface reduces drop mobility. Reduced drop mobility is more prominent for drop sizes that are comparable to the tube diameter and is maximum when mass transfer to and from the interface is inhibited. As the fractional coverage of soluble surfactants, or the interfacial Peclet number increases, larger Marangoni stresses are generated along the interface that lead to greater retardation of the drop motion.
\end{abstract}

Keywords: buoyancy-driven; drop motion; tube flow; surfactants; Marangoni stress; inertial effects

\section{Introduction}

The motion of deformable drops through a tube is of interest in a variety of industrial and technical applications such as oil recovery and production of pharmaceuticals, food and cosmetics and has been studied extensively [1]. In the creeping flow limit, several studies have theoretically, experimentally, and numerically determined the terminal velocity of buoyancydriven motion of drops and bubbles in cylindrical tubes [2, 3, 4, 5, 6, 7, 8]. Bretherton [2] found that the drop would rise only if Bond number, $B o=\left(\Delta \rho g R_{t}^{2} / \sigma\right)$, was greater than 0.842 , where $\Delta \rho$ was the difference in density between the two fluids, $R_{t}$ was the tube radius, and $\sigma$ was the interfacial tension between the two fluids. The rise velocity, $U$ of long bubbles was independent of bubble size, the film thickness was proportional to $\mathrm{Ca}^{2 / 3}$, and that there was a wave-like appearance (bulge) at the rear meniscus. Bretherton's analysis, however, was limited to vanishing values of $\mathrm{Ca}$ with the

${ }^{1}$ Corresponding Author: Tel.: +1 603862 3655; fax: +1 6038623747 .

E-mail address: ngupta@unh.edu (N.R. Gupta).

URL: http://pubpages.unh.edu/ ngupta/. 
error of about $10 \%$ for $\mathrm{Ca}=8 \times 10^{-5}$. The analysis was confirmed and extended to larger values of $\mathrm{Ca}$ for $0.0001 \leq \mathrm{Ca} \leq 0.1$ in the numerical work by Reinelt [7]. Borhan and Pallinti [8] experimentally obtained the velocity-volume curves for the buoyancy-driven motion of viscous drops through vertical cylindrical capillaries under creeping flow conditions for a wide range of viscosity ratios, density ratios, Bond numbers, and capillary numbers. As the Bond number increased, the drop became elongated, and the film thickness increased to a plateau value. For small Bond numbers, the terminal velocity as a function of drop size showed a local maxima due to the wall effect. For large Bond numbers, the terminal velocity increased monotonically with drop size. For both cases, the terminal velocity for large enough drop became independent of drop size. They also showed that the numerical solutions using the boundary integral method were in good agreement with the experimental measurements. They observed tip-streaming for large Bond numbers and at vanishing values of drop fluid viscosity compared to the bulk fluid viscosity and attributed it to the presence of surface-active impurities.

More recently, experiments $[9,10]$ as well as computational studies $[11,12,13,14]$ have been conducted to study the effects of inertia on the buoyancy-driven motion of drops and bubbles in tubes. Viana et al. [9] presented universal correlations to predict the terminal velocity of long bubbles in round pipes based on experimental data for wide ranges of tube buoyancy Reynolds numbers and Eotvos numbers. It was found that long bubble terminal velocity is independent of bubble volume. Li et al. [10] experimentally studied the buoyancy-driven motion of bubbles in circular and square channels over a range of Reynolds numbers at moderate capillary and Bond numbers. Bubbles in a circular channel were more prolate and rose slower than bubbles in a square channel with the same hydraulic diameter. As the Weber number increased, the bubbles developed a negative curvature at the rear end due to the increasing inertial effects. Bozzi et al. [11] numerically studied the buoyancy-driven motion of deformable drops at intermediate Reynolds numbers using a Galerkin finite-element method. For capillary numbers ranging from 0.005 to 1 , they found that the drops became oblate and even lost the rear convexity as inertia was increased by increasing Reynolds or Weber number. They observed novel hat-shaped drops. Taha and Cui [12] presented numerical studies to investigate the buoyancy-driven and pressuredriven motion of single Taylor bubbles (long bubbles) in vertical cylindrical tubes using FLUENT software implemented with volume-of-fluid (VOF) method. They showed that the bubble shape was found to be dependent upon ambient liquid viscosity and surface tension but not on the bubble size. Feng [13] used a Galerkin finite-element method with a boundary-fitted mesh to study the buoyancy-driven motion of a bubble in a round tube filled with a viscous liquid. Small bubbles exhibited similar behavior to bubbles moving in an infinite liquid and developed a spherical-cap when the Reynolds number was increased and the capillary number was not too small. Long bubbles exhibited a prolate nose-like shape with various tail shapes which could be adjusted by changing the Reynolds number and the Weber number. At large Weber numbers, $W e>10$, the long bubble formed a concave profile with a "cup" at small Reynolds number and a "skirt" with sharply curved rims at larger Reynolds number. For Weber numbers less than 1, the bubble tail appeared rounded without large local curvatures though a slightly concave tail developed at larger Reynolds numbers. Non-uniform annular film with a bulge forming at the rim of the bubble tail was observed for small Weber numbers suggesting the surface tension effect could play a role. Hayashi and Tomiyama [14] developed a drag correlation for fluid particles rising in a vertical pipe at low and intermediate Reynolds numbers using available drag correlations and simulations using a front tracking method. 
Surfactants are added deliberately or present as impurities in most multiphase systems and can affect the terminal velocity as well as deformation of the drops and bubbles. For buoyancydriven flow, surfactant molecules adsorbed on the interface convect from the front to the rear of the drop resulting in accumulation of surfactants at the rear stagnation point. Marangoni stresses that are generated due to the gradients in interfacial tension can significantly modify the viscous stresses resulting in reduced mobility of the drops. A number of studies have considered the effect of surfactants on the buoyancy-driven motion of drops and bubbles in a quiescent fluid and the pressure-driven motion of drops and bubbles in a tube. A relatively limited number of studies have considered the effect of surfactants on the buoyancy-driven motion of bubbles and drops in the creeping flow limit $[15,16]$ as well as at finite Reynolds number $[17,18,19]$.

Almatroushi and Borhan [15] experimentally studied the effect of surfactants on the steady shape and terminal velocity of drops and bubbles in a vertical tube with $3 \leq B o \leq 6.5$ in the creeping flow limit. They found that the presence of surfactants retarded the motion of small bubbles due to the development of adverse Marangoni stresses, while enhancing the motion of large bubbles by allowing them to deform away from the wall more easily. For viscous drops, the enhancement of mobility for large drops became more pronounced when increasing the surfactant concentration. Daripa and Pasa [16] studied the effect of surfactant on the terminal velocity and film thickness of a long bubble rising in a vertical capillary tube. Using analytical tools they showed that in the presence of surfactants, the rise velocity of the bubble are reduced compared to surfactant-free bubbles. Tasoglu et al. [17] used a finite-different/front-tracking method to study the motion of buoyancy-driven bubbles in the presence of soluble surfactants at finite Reynolds numbers for bubbles that were small compared to the tube diameter. They showed that the surfactants generally reduced the terminal velocity of the bubbles and this effect was most pronounced for the nearly spherical bubbles. The retardation effect of surfactants also made the contaminated bubble behavior more like a solid sphere. Hayashi and Tomiyama [18] studied the effect of soluble surfactants on the rise velocity of long bubbles in vertical tubes using an interface tracking method for very low Morton number and intermediate Eotvos numbers. They found that the surfactant does not affect terminal velocity of large Eotvos number bubbles as surface tension is negligible for such bubbles. Kurimoto et al. [19] assessed the applicability of the available drag correlations by experimentally and numerically studying the shapes and terminal velocities of drops with varying viscosity ratios in vertical pipes. They considered surfactant-free drops as well as drops in surfactant solutions above the critical micellar concentration. They found that the effect of surfactants on the drop shape and terminal velocity became significant as the Eotvos number was reduced or the viscosity ratio was increased.

In this study, we focus on the steady deformation and mobility of buoyancy-driven drops in a tube ranging in size from $0.2-1.3$ of tube radius at finite Reynolds numbers. We use a fronttracking method where both the drop and bulk phases are treated as incompressible Newtonian fluids. We further investigate the effect of surfactant mass transfer and coverage on the drop mobility for the range of drop sizes. Surfactants are modeled using a Langmuir equation of state in the adsorption-desorption limit

\section{Problem formulation}


The system consists of an axisymmetric viscous drop of density $\rho_{1}$ and viscosity $\mu_{1}$ rising through a quiescent immiscible viscous liquid of density $\rho_{2}$ and viscosity $\mu_{2}$ in a cylindrical tube of radius $R_{t}$ as shown in Figure 1. Both phases are treated as incompressible fluids. The gravitational vector $\mathbf{g}=-g \mathbf{e}_{z}$, points in the negative $z$-direction. The drop migration velocity is denoted as $U_{T}^{*}$ along the axis of the channel. In the following mathematical description, the reference frame is attached to the center of mass of the rising drop so that the tube wall moves downwards relative to the drop with the drop migration velocity. The drop size is characterized by the radius of a spherical drop of the same volume, $a=\left(3 V_{d} / 4 \pi\right)^{1 / 3}$ where $V_{d}$ is the volume of the drop.

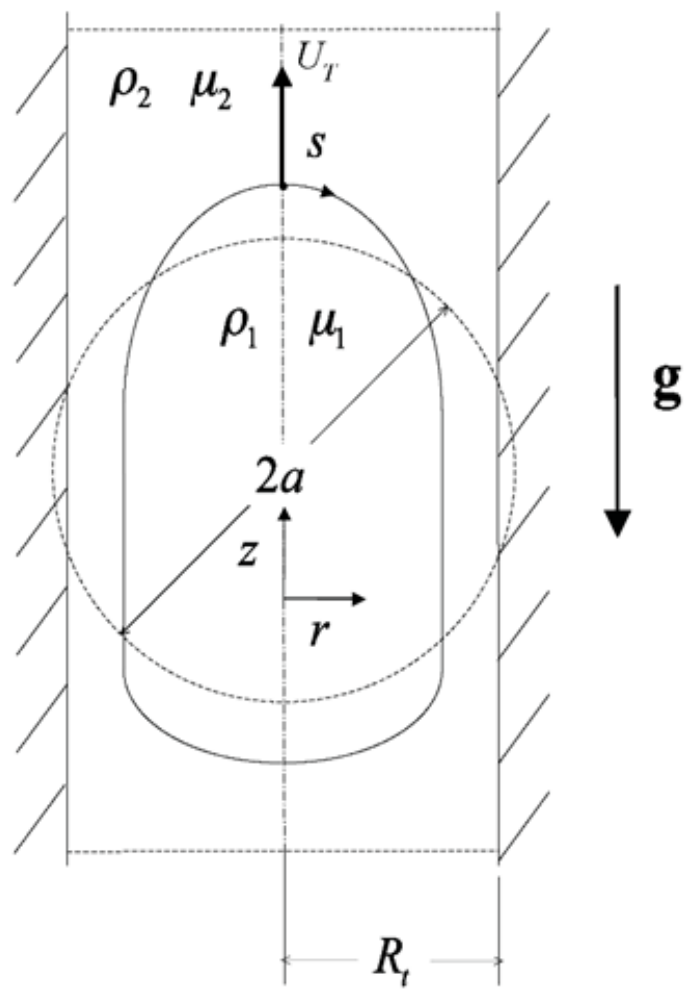

Figure 1: Schematic of the drop rising process through a quiescent immiscible viscous liquid.

We non-dimensionalize all lengths with the tube radius $R_{t}$, all velocities with the characteristic velocity $U_{c}=\left(\rho_{2}-\rho_{1}\right) g R_{t}^{2} / \mu_{2}$, and the pressure with $\mu_{2} U_{c} / R_{t}$. The dimensionless drop size is defined as, $\kappa=a / R_{t}$. The dimensionless velocity and pressure fields in each phase are governed by the incompressible Navier-Stokes equations given by,

$$
\nabla \cdot \mathbf{u}_{i}=0
$$




$$
\rho \operatorname{Re}\left(\frac{\partial \mathbf{u}_{i}}{\partial t}+\mathbf{u}_{i} \cdot \nabla \mathbf{u}_{i}\right)=-\nabla P+\nabla \cdot \tau
$$

Here, $P$ represents the modified pressure given by $p_{i}+\rho_{i} g z-\rho_{i}\left(d U_{T} / d t\right) z$ where $p$ denotes the pressure and $d U_{T} / d t$ gives the acceleration of the reference frame which goes to zero when steady state is reached. $\tau$ represents the shear stress in the Newtonian fluid and is given by $\boldsymbol{\tau}=\mu\left[\left(\nabla \mathbf{u}_{i}\right)+\left(\nabla \mathbf{u}_{i}\right)^{T}\right]$. The subscript $i=1$ or 2 depending on whether the inner fluid or the outer fluid is considered and $R e=\rho_{2} U_{c} R_{t} / \mu_{2}$ is the Reynolds number. In Eq.(2), $\rho=1+(\chi-1) \delta_{1 i}$, and $\mu=1+(\lambda-1) \delta_{1 i}$ with $\chi=\rho_{1} / \rho_{2}$ and $\lambda=\mu_{1} / \mu_{2}$ representing the density and viscosity ratio, respectively.

No-slip and impermeable boundary conditions are imposed at the solid tube walls and symmetry boundary conditions are imposed at the central axis $(r=0)$. At the far-field upstream and downstream boundaries, zero velocity and pressure gradients are specified. At the interface, continuity of velocity and the kinematic condition, $d \mathbf{x}_{s} / d t=\mathbf{u}_{s}$, are imposed. Here $\mathbf{x}_{s}$ is the location of the interface, $\mathbf{u}_{s}=u_{n} \mathbf{n}+u_{t} \mathbf{t}$ is the interfacial velocity, $u_{n}$ and $u_{t}$ are the normal and tangential components, and $\mathbf{n}$ and $\mathbf{t}$ are the outward pointing unit normal and unit tangent vectors at the interface. The stress jump across the interface is balanced by the Laplace pressure normal to the interface, and the Marangoni stress tangent to the interface,

$$
\left(P_{1}-P_{2}\right) \mathbf{n}+\left(\boldsymbol{\tau}_{2}-\boldsymbol{\tau}_{1}\right) \cdot \mathbf{n}=\frac{1}{C a}\left[\sigma \mathbf{n}(\nabla \cdot \mathbf{n})-\nabla_{s} \sigma\right]-\left[\frac{B o}{C a}-(1-\chi) \frac{d U_{T}}{d t} R e\right] z \mathbf{n}
$$

where $\nabla_{s}=(\mathbf{I}-\mathbf{n n}) \cdot \nabla$ is the interfacial gradient operator and $\sigma$ is the interfacial tension made dimensionless with a characteristic interfacial tension, $\sigma_{c}$. For surfactant-free simulations, $\sigma_{c}=\sigma_{o}$ where $\sigma_{o}$ is the clean interfacial tension, while for surfactant systems, $\sigma_{c}=\sigma_{e q}$ where $\sigma_{e q}$ is the equilibrium interfacial tension. The Bond number, $B o=\left(\rho_{2}-\rho_{1}\right) g R_{t}^{2} / \sigma_{c}$ gives the relative significance of gravitational forces to interfacial forces. Due to the selected characteristic velocity $U_{c}$, the capillary number, $\mathrm{Ca}=\mu_{2} U_{c} / \sigma_{c}$ which gives the ratio of viscous to interfacial forces becomes equal to the Bond number so that Eq. (3) can be simplified as

$$
\left(P_{1}-P_{2}\right) \mathbf{n}+\left(\boldsymbol{\tau}_{2}-\boldsymbol{\tau}_{1}\right) \cdot \mathbf{n}=\frac{1}{B o}\left[\sigma \mathbf{n}(\nabla \cdot \mathbf{n})-\nabla_{s} \sigma\right]-\left[1-(1-\chi) \frac{d U_{T}}{d t} \operatorname{Re}\right] z \mathbf{n}
$$

If there are no surfactants present in the system, interfacial tension $\sigma$ is uniform along the interface. When surfactants are present in the system, they can lower the interfacial tension where they accumulate. In the dynamic process of drop rising, surfactants adsorb and move along the interface and $\sigma$ then depends on the local surfactant interfacial concentration $\Gamma$. In this study, the surfactant present with bulk concentration $C_{\infty}$ is assumed to adsorb on the interface to an equilibrium surface concentration $\Gamma_{e q}$ according to the Langmuir adsorption 
isotherm [20]. Combining that with the Gibbs adsoption equation at constant temperature which relates the bulk concentration to the interfacial tension, the Langmuir equation of state is recovered. Its dimensionless form is given by,

$$
\sigma=\frac{1+E \ln (1-x \Gamma)}{1+E \ln (1-x)}
$$

where the interfacial tension $\sigma$ and the surfactant interfacial concentration $\Gamma$ are made dimensionless with the equilibrium interface tension, $\sigma_{e q}$ and the equilibrium surfactant concentration $\Gamma_{e q}$ respectively. In Eq. (5), $E=R T \Gamma_{\infty} / \sigma_{o}$ is the elasticity parameter which represents the sensitivity of the interfacial tension to the surfactant concentration and $\Gamma_{\infty}$ is the maximum monolayer packing of surfactants. The initial interfacial coverage of surfactants, $x=\frac{\Gamma_{e q}}{\Gamma_{\infty}}=\frac{\beta C_{\infty} / \alpha}{1+\left(\beta C_{\infty} / \alpha\right)}$ where $\beta$ and $\alpha$ represent the kinetic constants for surfactant adsorption and desorption respectively. The Langmuir equation of state captures the nonlinear behavior of surfactants where the interfacial tension reduces sharply as the interfacial concentration of surfactants approaches the maximum packing limit. This allows us to accurately describe interfacial tension for immiscible flow systems where convection leads to regions of high surfactant concentration locally.

As the interface deforms, the surfactant concentration at the interface, $\Gamma(s, t)$, depends on the interface location, $s$, and time, $t$, and is determined by the interfacial mass balance. Surfactants are transported by convection and diffusion along the interface, as well as by adsorptiondesorption and diffusion between the interface and the bulk. When surfactants are transported between interface and bulk as fast as they are transported along the interface, and the adsorptiondesorption rate is much lower than diffusion rate between interface and bulk, we designate surfactants as adsorption-desorption controlled surfactants. This situation occurs when high bulk concentration of surfactants are supplied to the interface, the adsorption-desorption becomes the rate-limiting step, and the diffusion between the interface and bulk can be neglected. In this study, we consider the soluble surfactant case in the adsorption-desorption limit with the surfactant diffusivity, the kinetic constants for surfactant adsorption and desorption given by $D_{s}$, $\beta$, and $\alpha$ respectively. The dimensionless interfacial mass balance for the surfactants is given by [21, 22],

$$
\frac{\partial \Gamma}{\partial t}+\nabla_{s} \cdot\left(\Gamma u_{t} \mathbf{t}\right)+\Gamma(\nabla \cdot \mathbf{n}) u_{n}-\frac{1}{P e_{s}} \nabla_{s}^{2} \Gamma=\frac{B i}{1-x}(1-\Gamma) .
$$

Here the interfacial Peclet number, $P e_{s}=U_{c} R_{t} / D_{s}$ is the characteristic ratio of the interfacial convection rate to the interfacial diffusion rate and the Biot number, $B i=\alpha R_{t} / U_{c}$ is the characteristic surfactant desorption rate to the convection rate. The adsorption-desorption flux on the right-hand side of Eq. (6) assumes that surfactant adsorption is first-order in bulk concentration and the space available on the interface, and surfactant desorption is first order in interfacial concentration. 


\section{Solution Procedure}

We implement a hybrid volume-of-fluid (VOF) method with a front-tracking scheme [23, 20, $24,25,26,27$ ] since it combines the mass conservation properties of the VOF method [28] with the accuracy of defining the interface topology and stresses of the front-tracking method. The Navier-Stokes equations are solved using a VOF method on a fixed Eulerian grid, and the interface is tracked by a moving Lagrangian grid. A continuous surface force method [29] is adopted to express the interfacial stress jump with a interfacial delta function, $\delta_{s}$ which is 1 at the interface and 0 everywhere else. The Navier-Stokes equations over the whole domain is expressed in the single-fluid VOF formulation as

$$
\begin{aligned}
& \nabla \cdot \mathbf{u}=0 \\
& \bar{\rho} \operatorname{Re}\left(\frac{\partial \mathbf{u}}{\partial t}+\mathbf{u} \cdot \nabla \mathbf{u}\right)=-\nabla P+\nabla \cdot\left(\bar{\mu}\left[(\nabla \mathbf{u})+(\nabla \mathbf{u})^{T}\right]\right) \\
&+\frac{1}{B o}\left[\nabla_{s} \sigma-\sigma \mathbf{n}(\nabla \cdot \mathbf{n})\right] \delta_{s}+\left[1-(1-\chi) \frac{d U_{T}}{d t} \operatorname{Re}\right] z \mathbf{n} \delta_{s},
\end{aligned}
$$

in which $\bar{\rho}=1-(1-\chi) \phi$ and $\bar{\mu}=1-(1-\lambda) \phi$. Here $\phi$ is the VOF function, which takes the value 1 for the drop phase and 0 for the bulk phase.

A time splitting method is used to solve the imcompressible Navier-Stokes equations for velocity and pressure fields. It separates the pressure Poisson equations and velocity equations by introducing an intermediate velocity field $\mathbf{u}^{*}$ that does not have to be divergence-free (refer to Eqs. (9) to (11)):

$$
\begin{aligned}
& \bar{\rho} \operatorname{Re}\left(\frac{\mathbf{u}^{*}-\mathbf{u}^{n}}{\Delta t}\right)=-\bar{\rho} \operatorname{Re}\left(\mathbf{u}^{n+1} \cdot \nabla \mathbf{u}^{n+1}\right)+\nabla \cdot\left(\bar{\mu}\left[\left(\nabla \mathbf{u}^{n+1}\right)+\left(\nabla \mathbf{u}^{n+1}\right)^{T}\right]\right) \\
&+\frac{1}{B o}\left[\nabla_{s} \sigma-\sigma \mathbf{n}(\nabla \cdot \mathbf{n})\right] \delta_{s}+\left[1-(1-\chi) \frac{d U_{T}}{d t} \operatorname{Re}\right] z \mathbf{n} \delta_{s}, \\
& \bar{\rho} \operatorname{Re}\left(\frac{\mathbf{u}^{n+1}-\mathbf{u}^{*}}{\Delta t}\right)=-\nabla P^{n+1}
\end{aligned}
$$

To ensure that $\mathbf{u}^{n+1}$ is divergence-free, i.e $\nabla \cdot \mathbf{u}^{n+1}=0$, by taking the divergence of Eq. (10),

$$
\bar{\rho} \operatorname{Re}\left(\frac{-\nabla \cdot \mathbf{u}^{*}}{\Delta t}\right)=-\nabla^{2} P^{n+1}
$$


Here, $n$ and $n+1$ is the successive time steps. The governing equations are discretized using a second-order accurate finite volume method on the fixed Eulerian grid with staggered representation of the velocity and pressure fields. We use previous velocities $\mathbf{u}^{n}$ to get $\mathbf{u}^{*}$ from Eq. (9), and then we solve the pressure Poisson equation (Eq. (11)) to get pressure fields. Finally, the new divergence-free velocity field can be obtained from Eq. (10). In order to calculate the advection and diffusion terms implicitly in Eq. (9), Eqs. (9)-(11) are solved iteratively at each time step to obtain $\mathbf{u}^{n+1}$ and $P^{n+1}$.

The interface is represented by Lagrangian marker particles with a parameter representation $(r(s), z(s))$ where $s$ is the arc length starting from the apex of the drop. For the initial shape, we assume a spherical drop shape if dimensionless drop size $\kappa$ is less than a prespecified value (e.g. 0.9), and a cylinder with two hemispherical end caps otherwise. The VOF function $\phi$ and the continuous surface force are calculated in the entire domain. The Navier-Stokes solver is used to calculate the velocity and pressure fields on the fixed grid using Eq. (8). The velocities of the marker particles on the moving grid at the interface are determined by employing a linear interpolation based on the velocity fields obtained on the fixed grid. The marker particles are advected as material particles based on the kinematic condition to determine the new interface location using an explicit Euler scheme. As the interface continuously deforms, marker particles are added or removed at each time step to maintain the same level of discretization. A volume correction technique proposed by Ryskin and Leal [30] is adopted to preserve the drop volume. We define a scaling factor $\beta_{v}=V^{n} / V^{0}$ where $V^{n}$ denotes the drop volume updated according to kinematic condition at nth time step and $V^{0}$ denotes the initial drop volume. Once the updated drop shape is obtained, we can calculate new $\phi$ field for next time step. In the presence of surfactants, the surfactant concentration, $\Gamma(s)$ and interfacial tension, $\sigma(s)$ are defined at each marker particle location. Starting with an equilibrium concentration of surfactants, $\Gamma=1$, the surfactant concentration and interfacial tension are updated using Eqs. (5) and (6). The interface location, surfactant concentration and interfacial tension are represented by cubic spline functions at any given time step. All surface derivatives in Eq (6) are calculated from their cubic spline representations. The mass balance, Eq. (6) is solved using a finite difference formulation. This algorithm is repeated until drop shape and terminal velocity reach the steady state. In the current simulations, the physical domain has a unit length in the radial direction and an axial domain length of 16 units was chosen to avoid any edge effects. The Eulerian and Lagrangian mesh sizes and time step are chosen to ensure convergence of drop shape, velocity and pressure fields for a chosen set of non-dimensional parameters. For most of the simulation results presented in this paper, a mesh size of at least 0.025 ( 40 cells per unit length) and a time step of at least $10^{-4}$ is used. Higher resolution of the mesh size (up to 0.0125 ) was used for larger drop sizes at high Bond numbers where thin films were seen. This algorithm was proven to efficiently simulate two-phase flows with deformable interface in our previous studies [26] [27].

\section{Results and Discussion}

The drop rising process is studied by running the simulations for a range of drop sizes, $0.1 \leq \kappa \leq 1.3$ for a specified set of dimensionless parameters such as Reynolds number, Bond number and so on. The simulations are run till a steady drop shape and a terminal velocity, $U_{T}$, 
are obtained. The velocity-volume curves are generated by plotting the terminal velocity, $U_{T}$ as a function of drop size, $\kappa$. In addition to the drop images, the drop shape is quantified by the deformation parameter, $\Delta \equiv(L-B) /(L+B)$, where $L$ and $B$ are the maximum axial and radial dimension of the drop at steady state. For a spherical drop shape, $\Delta=0$ while $\Delta>0$ for a prolate shape and $\Delta<0$ for an oblate shape. For the case of long drops, only the central region is chosen to measure the maximum radial length. Results are also presented in terms of the film thickness, $\delta$, defined as the dimensionless minimum distance between the drop interface and the capillary wall. For the case of long drops, film thickness in the central uniform region is reported.

\subsection{Validation Studies}

In order to validate the numerical scheme for drops rising in a confined tube, the numerical results in this study are compared with the experimental results of Borhan and Pallinti [8] where a viscous drop rises in a vertical precision-bore glass capillary. The experimental results used for comparison were for a suspending fluid of $84.2 \mathrm{wt} \%$ glycerol-water mixture with density and viscosity of $1212 \mathrm{~kg} / \mathrm{m}^{3}$ and $80 \mathrm{mPa} \cdot \mathrm{s}$, respectively. The drop fluid was a Dow Corning silicone fluid (DC510-100) with a density and viscosity of $990 \mathrm{~kg} / \mathrm{m}^{3}$ and $105 \mathrm{mPa} \cdot \mathrm{s}$, respectively. The interfacial tension between the two phases was $26.8 \times 10^{-3} \mathrm{~N} / \mathrm{m}$. Based on the reported experimental data, the dimensionless parameters for the numerical simulations are set to $\chi=0.82, \lambda=1.3, \operatorname{Re}=26$, and $B o=1.3$. A comparison of steady shapes for drops of size $\kappa=$ $0.58,0.73,0.92,1.15$, and 1.32 is shown in Figure 2(a). Since no scale was available for the experimental data, we scaled our simulation for a drop size of $\kappa=0.58$ to match the experimental shape for the same drop size. We then scaled all the remaining simulations based on the scaling obtained for $\kappa=0.58$ and superimposed them on the experimental shapes from Figure 6 in [8]. It must be noted that for the experimental shapes, the edges of the image did not correspond to the edge of the tube wall. There is good qualitative agreement between the numerical and the experimental results for drop shapes with small drops being nearly spherical. As the drop size increases, the width of the drop approaches an upper bound and does not change much with any further increase in the drop size. The drop loses fore and aft symmetry with a higher positive curvature at the front of the drop. For very large drop sizes, a central region with a constant film thickness, similar to a Bretherton bubble is observed. The terminal velocities of the steady drop shapes obtained numerically are also compared to the experimental terminal velocities in Figure 2(b). There is good quantitative agreement between the experimental and numerical terminal velocities. As the drop size increases, the terminal velocity decreases due to the increased wall drag and it eventually reaches a constant value which is independent of the drop size. The experimentally observed terminal velocities were consistently lower than the numerically predicted velocities. Even a small amount of surfactant present in the experiments would result in reduced mobility of drops and may be a possible explanation for the observed difference. 

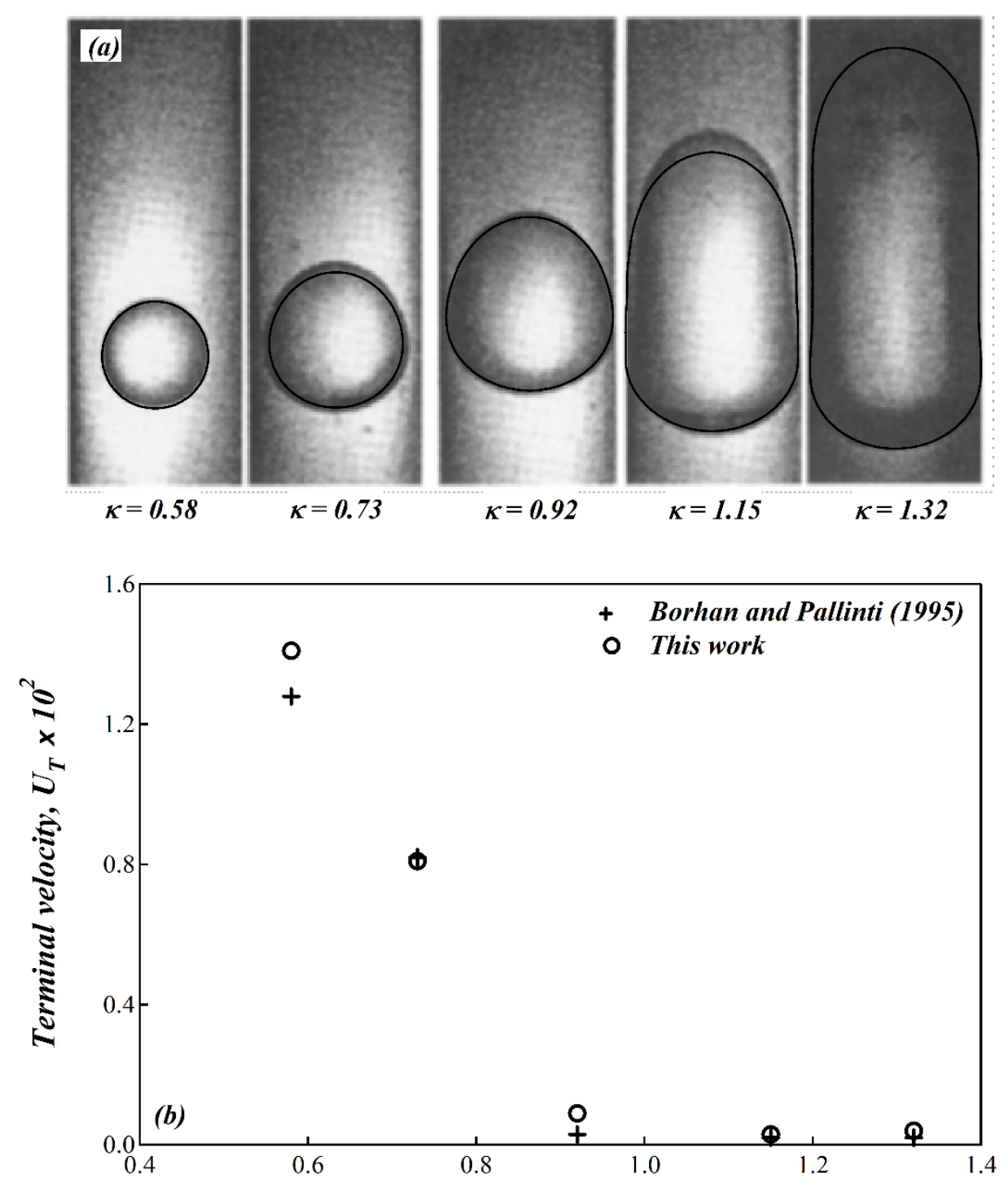

Drop size, $\kappa$

Figure 2: Comparison of (a) steady drop shapes and (b) terminal velocity as a function of drop size observed in Borhan and Pallinti's [8] experimental work and predicted in our numerical simulation.

\subsection{Surfactant-free Drops}

For the surfactant-free case, we present the effect of Bond number, Bo and the Weber number, $W e_{T}$ on the steady drop deformation and mobility while holding $\lambda=0.1, \chi=0.1$, and $\operatorname{Re}=10$. We choose $\lambda<1$ as surfactant effects are more dominant when the viscosity of the drop phase is low. The effect of Bond number on the steady drop velocity as a function of drop size is seen in Figure 3. For $B o=1$, the terminal velocity of the drops first increases with increasing drop size due to predominantly buoyancy effects. As the drop size becomes comparable to the tube size, the terminal velocity of the drop decreases due to increased wall drag and eventually reaches a constant value which is independent of the drop size. For the simulations of drops with $B o=10$, 
the terminal velocities of drops with $\kappa \leq 0.6$ show a maxima in the terminal velocity at $\kappa=0.5$ similar to the $B o=1$ curve. However, the magnitude of the terminal velocity at $B o=10$ is smaller than the terminal velocity of a $B o=1$ drop of the same size. For $\kappa>0.6$, the terminal velocities start to increase again reaching a plateau value much higher than $B o=1$ drops. Borhan and Pallinti [8] also experimentally observed a decrease in the retarding effect of the capillary wall with an increasing Bond number.

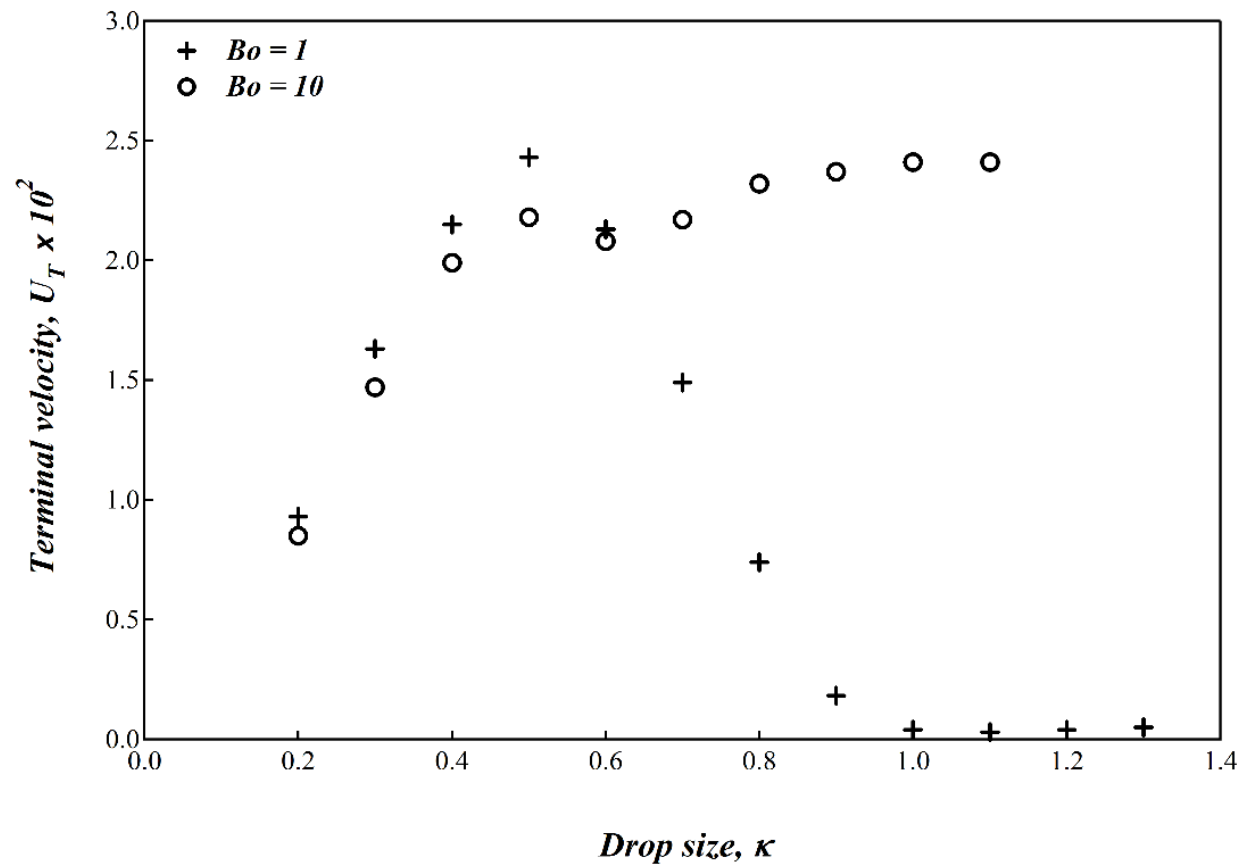

Figure 3: Comparison of terminal velocity of the drop as a function of drop size for $B o=1$ and 10 for $\chi=\lambda=0.1$ and $R e=10$.

The Bond number represents the relative significance of the gravitational force which tends to deform the drop and the interfacial force that opposes this deformation. For these simulations with different Bond numbers, the gravitational force is the same as the density ratio is held constant at $\chi=0.1$. So, a larger Bond number indicates lower interfacial tension and hence a lesser resistance to deformation. A comparison of steady drop shapes, the deformation parameter, and the film thickness as a function of drop size for $B o=1$ and $B o=10$ are shown in Figures 4 and 5. For high interfacial tension drops with $B o=1$, the length and width of small drops increases almost linearly with drop size and the drop stays nearly spherical for $\kappa<0.9$. The deformation parameter, thus, remains almost zero for $\kappa<0.9$ and the film thickness reduces linearly with increasing drop size as seen in Figure 5. For $\kappa>0.9$, the width of the drop approaches an upper bound that does not change much with increasing drop size while the length of drop increases fast making the drop more and more prolate. This results in a near linear increase in the deformation parameter and a constant film thickness for $\kappa>0.9$. As lower interfacial tension drops with $B o=10$, the drop shapes are still nearly spherical for small drop sizes, $\kappa \leq 0.6$, with negligible wall effect though they lose their fore and aft symmetry as seen in Figure 4. For larger drops with $\kappa>0.6$, the drops with a larger Bond number are more prolate 
(deformed) as expected resulting in a larger film thickness as seen in Figure 5. The more prolate shape of the drops at higher Bond numbers results in larger terminal velocities than lower Bond number drops.

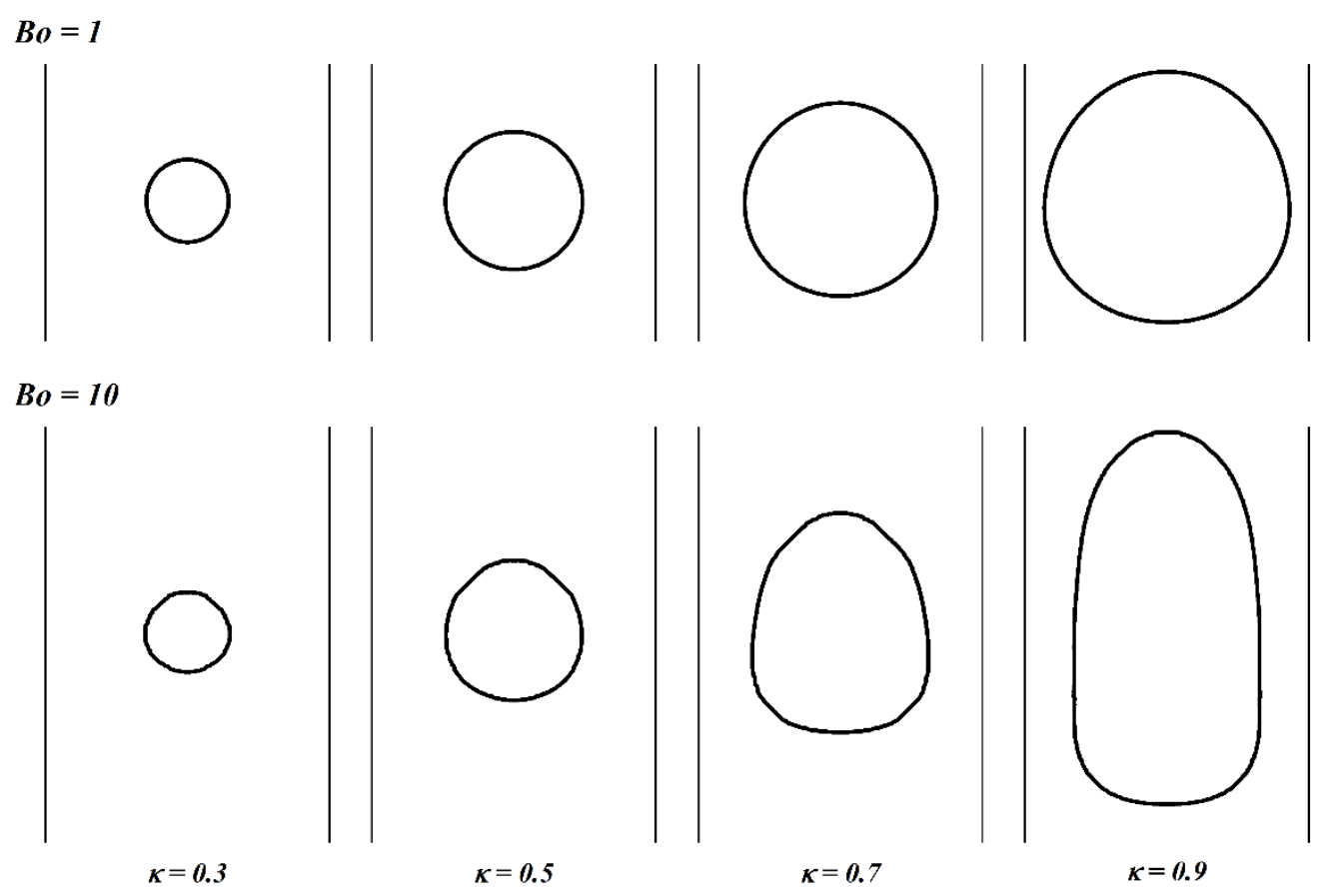

Figure 4: Effect of Bond number on the steady drop shapes for $\chi=\lambda=0.1$ and $R e=10$.

The inertial effect is captured by the Weber number, $W e_{T}$, which is defined as $W e_{T}=\rho U_{T}^{2} R_{t} / \sigma_{c}$ and is a measure of the inertial force as compared to the interfacial force. Since the Weber number is based on the drop terminal velocity, it cannot be set for a given simulation. For a chosen set of dimensionless numbers, $\lambda, \chi, R e, B o$, and size $\kappa$, the terminal velocity, $U_{T}$ and the Weber number, $W e_{T}$ is determined after the drop reaches a steady state. To study the effect of Weber number, we set $\chi=\lambda=0.1$ and vary Reynolds and Bond numbers simultaneously while keeping the ratio of $R e / B o=10$. The steady drop shapes and the corresponding Weber numbers for intermediate drop sizes $(\kappa=0.5)$ as well as large drop sizes $(\kappa=1)$ are shown in Figure 6. As the Weber number is increased, drop deforms, becomes flat at the rear and eventually develops a negative curvature. The flattening and development of negative curvature at the drop rear have been observed for both small and large drops with increasing Weber numbers in the experimental work by Li et al. [10]. 

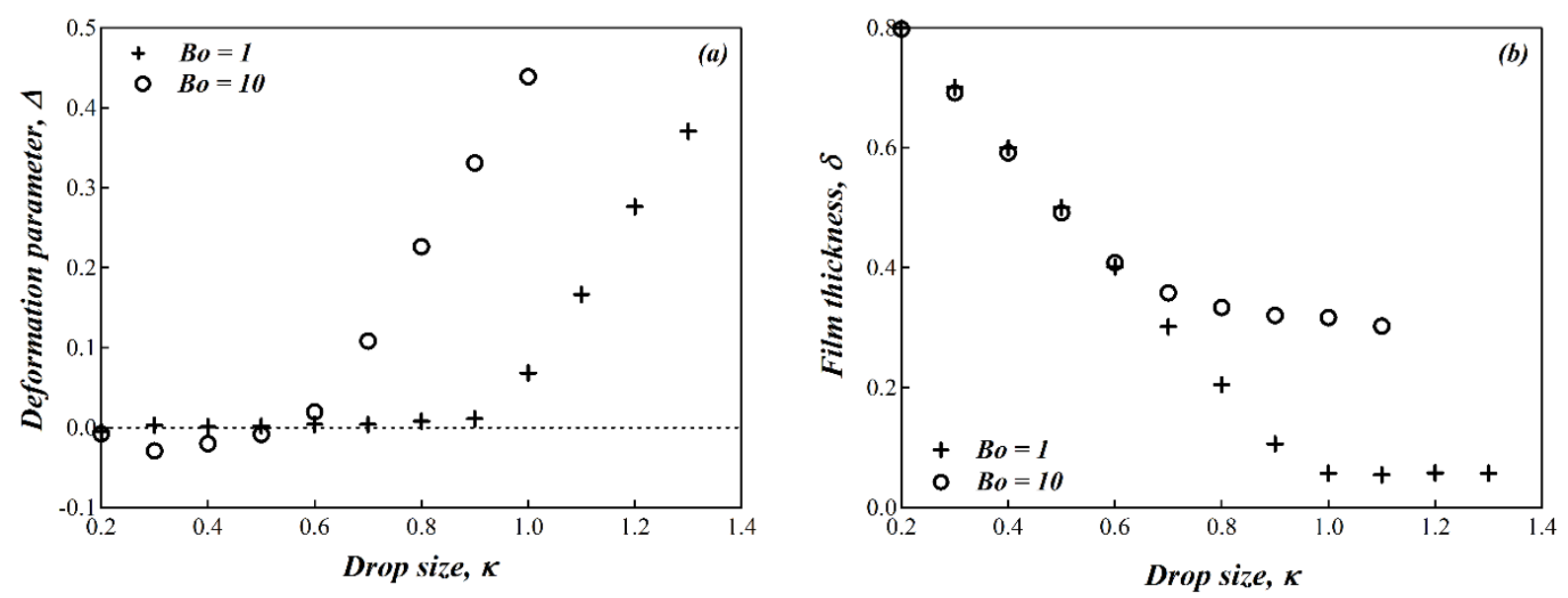

Figure 5: Comparison of the (a) deformation parameter and (b) film thickness of the rising drop at steady state as a function of drop size for $B o=1,10$ for $\chi=\lambda=0.1$ and $R e=10$.

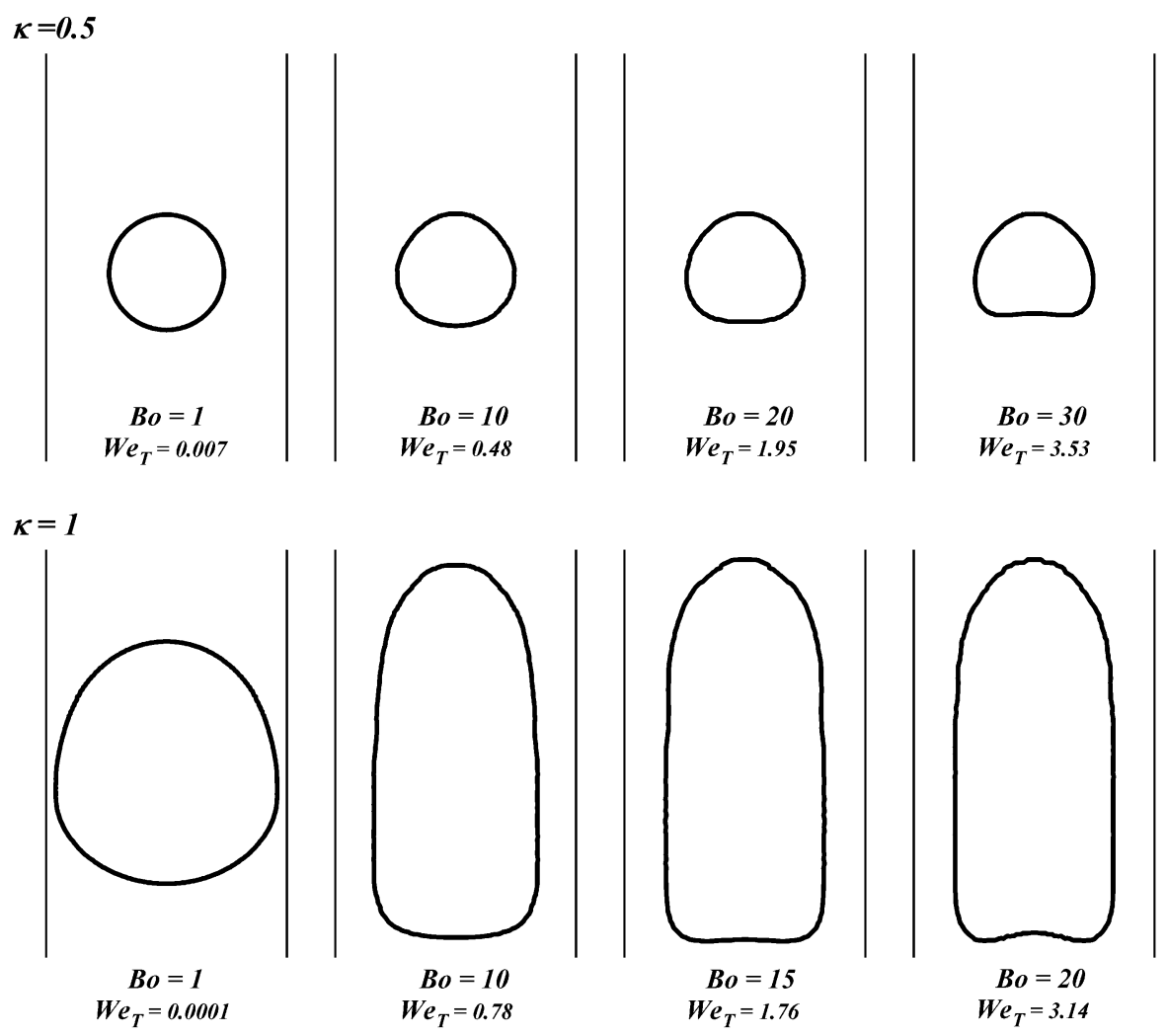

Figure 6: The steady drop shapes for both small drop size $(\kappa=0.5)$ and large drop size $(\kappa=1)$ with varying Weber number for $\chi=\lambda=0.1$, and $R e / B o=10$.

\subsection{Surfactant-laden Drops}

For a drop rising in a tube, the tangential velocity along the interface is from the front of the drop (arclength, $s=0)$ to the rear of the $\operatorname{drop}\left(s=s_{\max }\right)$. If surfactants are present at the interface, the tangential flow along the interface sweeps surfactants from the front causing surfactant 
concentration to fall below the equilibrium value. Surfactants accumulate at the rear of the drop resulting in surfactant concentration to exceed the equilibrium concentration here. Surfactants are supplied by adsorption / desorption from the surfactant sublayer near the drop which is always maintained at the constant bulk concentration. In this section, we present the results for the effect of Biot number, interfacial coverage, and surface Peclet number on the steady deformation and velocity of drops with $\chi=\lambda=0.1, \operatorname{Re}=10, B o=1$. The elasticity number, $E$, gives the sensitivity of the interfacial tension to the variations in surfactant concentration. For all the simulations presented here, the elasticity number is chosen to be 0.164 as the typical value for most surfactant systems lies between 0.1 and 0.2 [31].
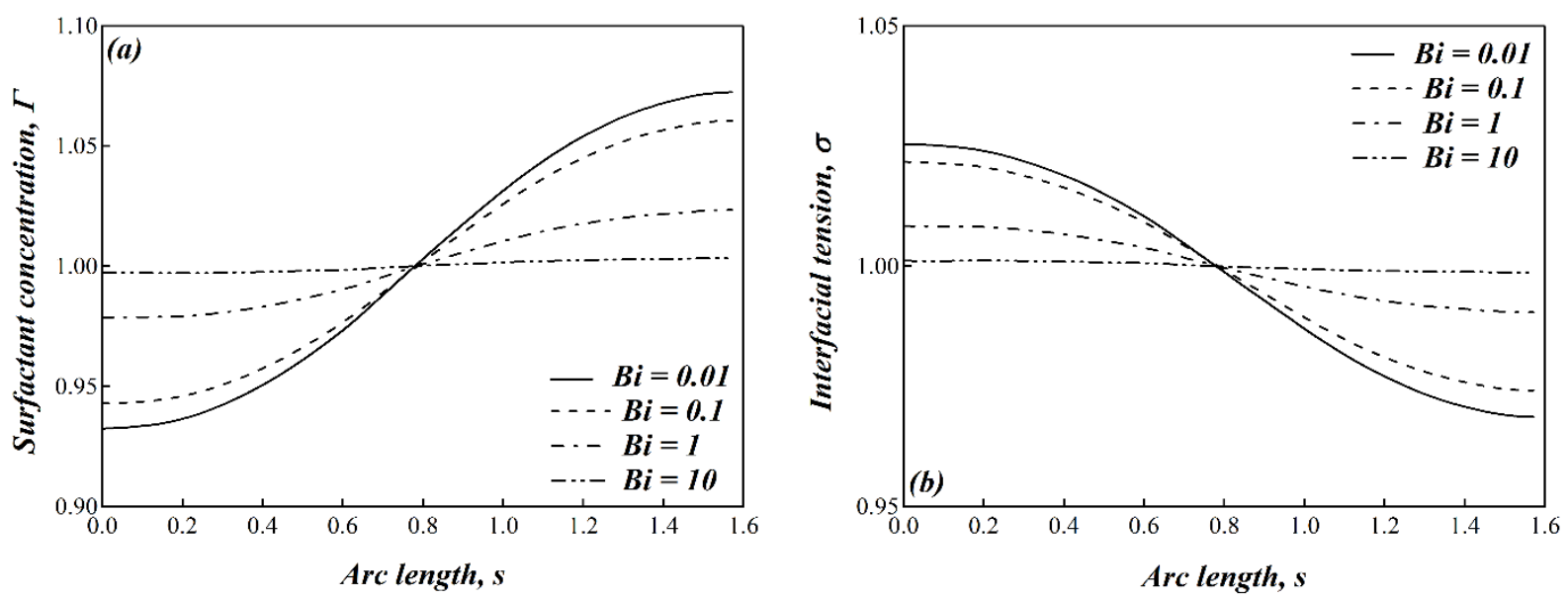

Figure 7: Comparison of (a) surfactant concentration and (b) interfacial tension along the interface at steady state for varying $B i$ for $\kappa=0.5$ drops with $\chi=\lambda=0.1, R e=10$, $B o=1, x=0.667$, and $P e_{s}=10$.

\subsubsection{Effect of Biot Number}

Surfactant mass transfer to and from the bulk compared to the convection flux along the interface, captured by the Biot number, plays an important role in surfactant distribution at the interface and hence its mobility. In the limit when surfactant mass transfer to the interface is much slower than surface convection, that is $B \boldsymbol{i}=0$, surfactants cannot adsorb/desorb from the interface and behave as insoluble surfactants. This leads to large surfactant concentration gradients and consequently interfacial tension gradients along the interface. The effect of Biot number on the surfactant concentration and interfacial tension along the interface of $\kappa=0.5$ drops with $\chi=\lambda=0.1, R e=10, B o=1, x=0.667$, and $P e_{s}=10$ is seen in Figure 7. The interfacial tension at the rear of the drop $\left(s=s_{\max }\right)$ is much lower than the front of the drop $(s=0)$ indicating the accumulation of surfactants at the rear of the drop due to the interfacial flow. As the Biot number increases, surfactants desorb from the rear of the drop where surfactant concentration is higher than the equilibrium while surfactants will adsorb at the front of the drop thereby reducing the interfacial tension gradients as seen in Figure 7. 


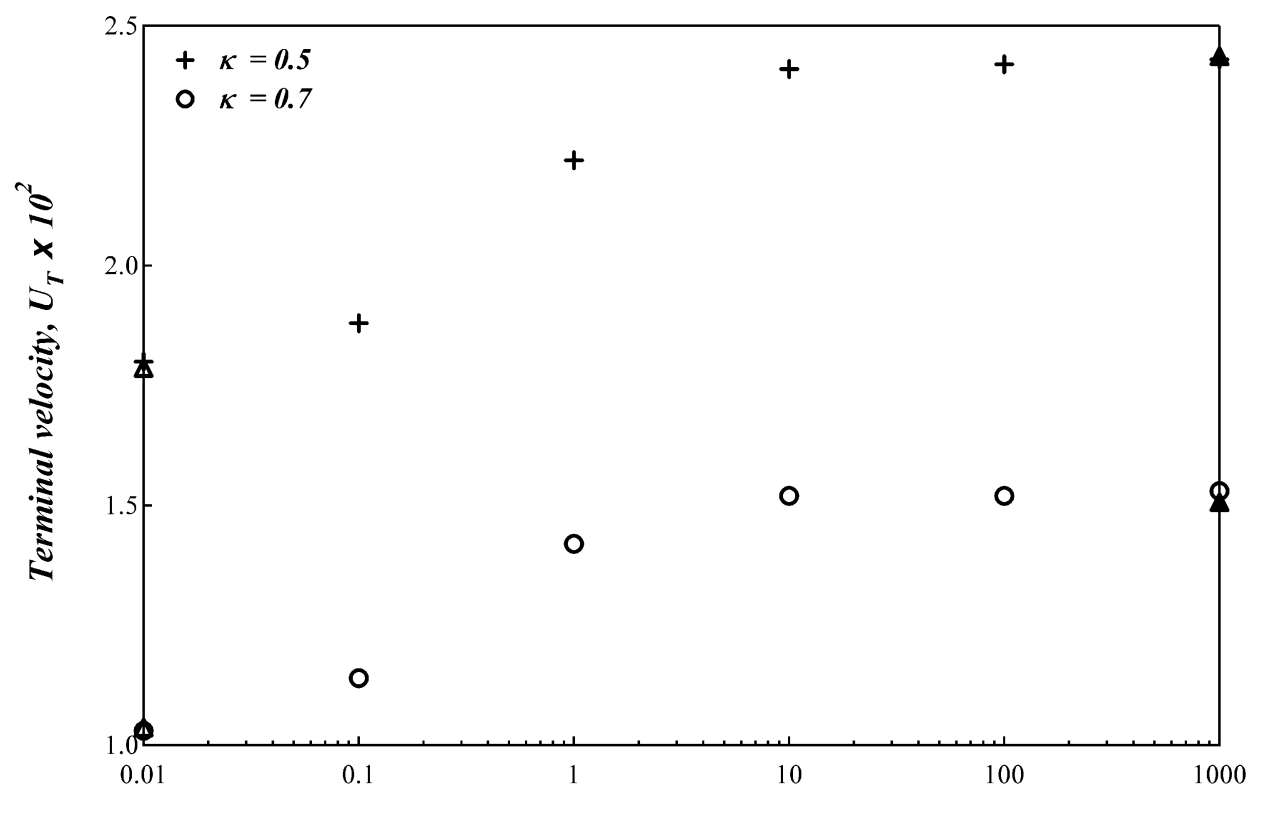

Biot number, $B i$

Figure 8: Terminal velocity of drops as a function of Biot number for $\kappa=0.5$ and 0.7 drops with $\chi=\lambda=0.1, R e=10, B o=1, x=0.667$, and $P e_{s}=10$. The open triangles and solid triangles represent the limits for $B i=0$ and $B i \rightarrow \infty$ respectively.

The large variation of interfacial tension along the interface for $B i=0.01$ seen in Figure 7 gives rise to Marangoni stresses that oppose the tangential flow along the interface. As a result the mobility of the drop is retarded. The effect of varying Biot number on the terminal velocity of drops of two different sizes, $\kappa=0.5$ and 0.7 are seen in Figure. The open triangles represent the terminal velocities of $\kappa=0.5$ and 0.7 drops in the presence of insoluble surfactants, $B i=0$. The simulated results tend to this limit as $B i \rightarrow 0$. As Biot number is increased, the gradients in interfacial tension and hence Marangoni stresses are reduced and the interface becomes more mobile. In the limit when mass transport of surfactants to the interface is much faster than interfacial convection, that is $B i \rightarrow \infty$, the surfactant concentration at the interface remains almost at the equilibrium concentration. This results in a uniform reduction in interfacial tension, the equilibrium interfacial tension. Since we define the Bond number based on the equilibrium interfacial tension, $B i=\infty$ corresponds to a simulation of a clean drop represented as a solid triangle on Figure 8 for the two drop sizes. Our numerical results for large Biot numbers approach the clean drop limit as expected. The maximum variation in terminal velocity is observed for intermediate Biot numbers, $0.1 \leq B i \leq 1$. A comparison of terminal velocity as a function of drop size for $B i=0.1,1$, and $\infty$ for $\chi=\lambda=0.1, \operatorname{Re}=10, B o=1, x=0.667$, and $P e_{s}=10$ is shown in Figure. In the presence of surfactants, maximum retardation of drop mobility is seen for drops of intermediate size when the adsorption/desorption to the interface is slow compared to convection, that is $B i \rightarrow 0$. As mass transfer to the interface increases, drops of all sizes become remobilized due to reduced Marangoni stresses. Even though the drop 
mobility is significantly affected, the drop shapes are insensitive to the surfactant mass transfer except at very large drop sizes.

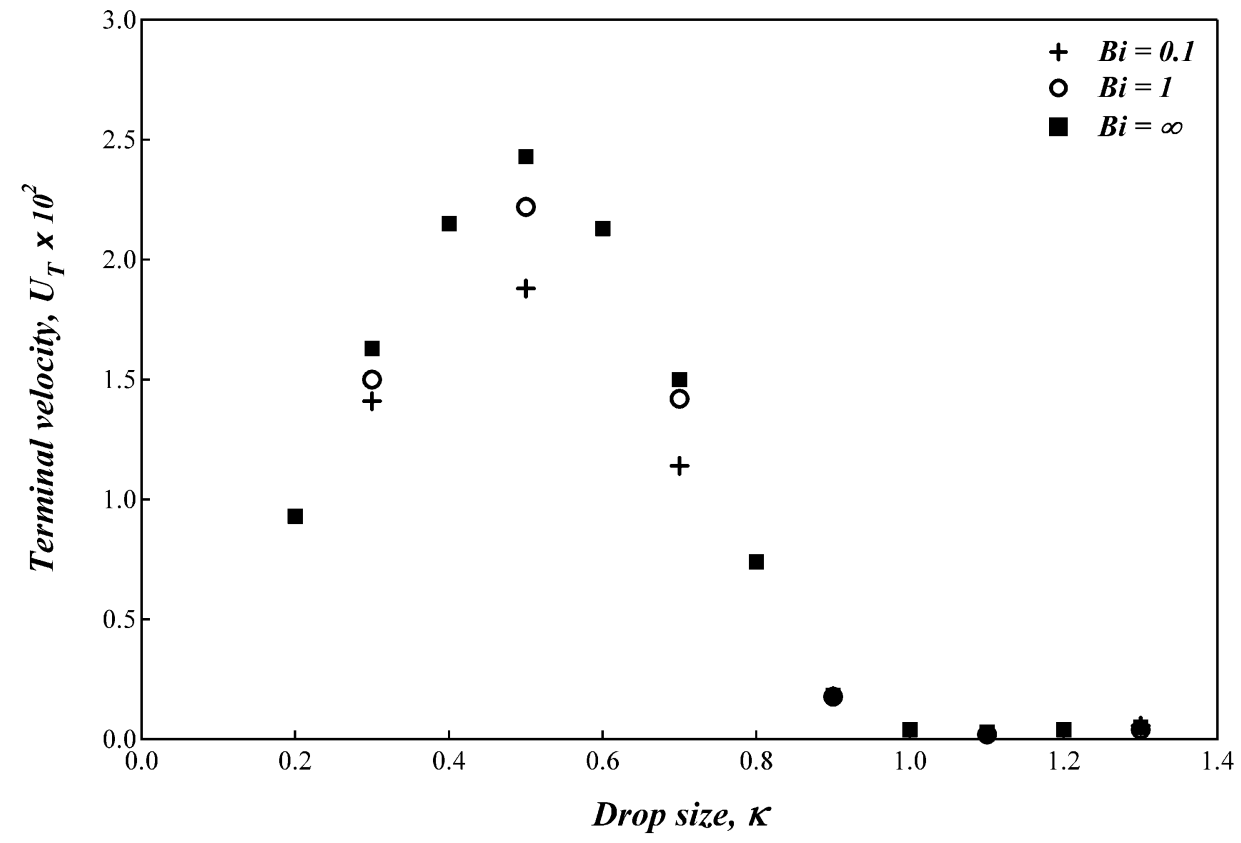

Figure 9: Comparison of terminal velocity as a function of drop size for $B i=0,0.1$, and $\infty$ for $\chi=\lambda=0.1, \operatorname{Re}=10, B o=1, x=0.667$, and $P e_{s}=10$.
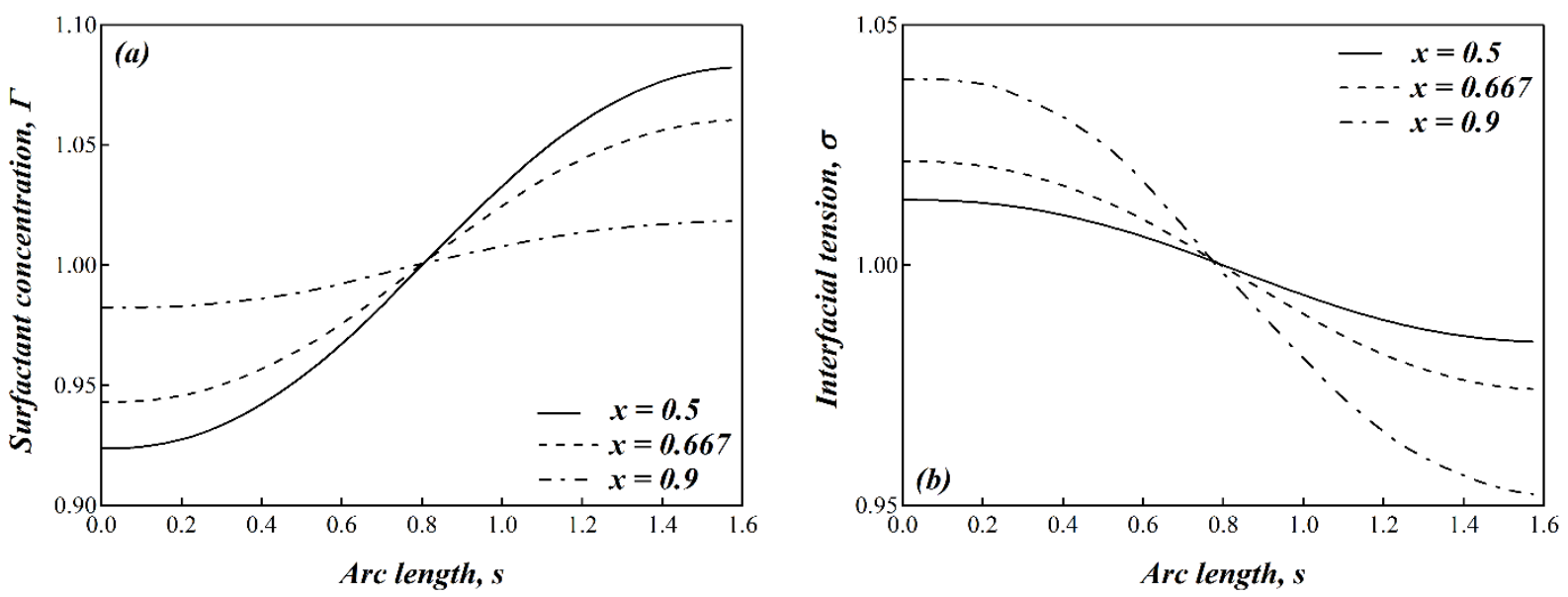

Figure 10: Effect of fractional coverage on the (a) surfactant concentration and (b) interfacial tension along the interface at steady state for $x=0.5,0.667$, and 0.9 for $\kappa=0.7$, $\chi=\lambda=0.1, R e=10, B o=1, B i=0.1$, and $P e_{s}=10$.

\subsubsection{Effect of Surfactant Coverage}


Next, we consider the effect of initial surfactant coverage, $x=\Gamma_{e q} / \Gamma_{\infty}$ on drop dynamics for $\chi=\lambda=0.1, R e=10, B o=1, B i=0.1$, and $P e_{s}=10$. For these simulations, since the equilibrium surfactant is held constant, increasing the initial surfactant coverage implies a reduction in the maximum packing, $\Gamma_{\infty}$. For small initial coverages $\left(\Gamma_{e q}<<\Gamma_{\infty}\right)$, large gradients in surfactant concentration are realized due to surface convection while the Marangoni stresses that are generated to oppose this flow are weak due to the weak coupling between the surface tension gradient, $\nabla_{s} \Gamma$ and $\nabla_{s} \sigma$. As the initial coverage increases $\left(\Gamma_{e q}\right.$ approaches $\Gamma_{\infty}$ from below), even small gradients in surfactant concentration result in large Marangoni stresses that oppose the tangential flow thereby reducing the gradients in surfactant concentration. The surfactant concentration and interfacial tension as a function of arc length for a $\kappa=0.7$ drop with different surface coverages is seen in Figure 10. As expected, with increasing values of initial surface coverage, the surfactant concentration gradients decrease while the interfacial tension gradients increase. The larger Marangoni stresses in the high initial surface coverage case results in a reduction in the terminal velocity of the drops. A comparison of the terminal velocity as a function of drop size for clean and $x=0.667$, and 0.9 drops shows the retardation in mobility especially for intermediate sized drops with increasing coverage. A comparison of steady drop shapes for clean and $x=0.9$ drops for $\kappa=0.5,0.7,0.9,1.1$, and 1.3 is shown in Figure 12. The drop shapes with surfactant coverage of $x=0.9$ are similar to the surfactant-free drops in that they stay spherical for small drops $(\kappa<0.7)$ but become more prolate for large drops $(\kappa \geq 0.7)$. As a consequence, the film thickness for large drops $(\kappa \geq 0.7)$ with soluble surfactants are higher than the corresponding film thickness in the absence of surfactants [15].

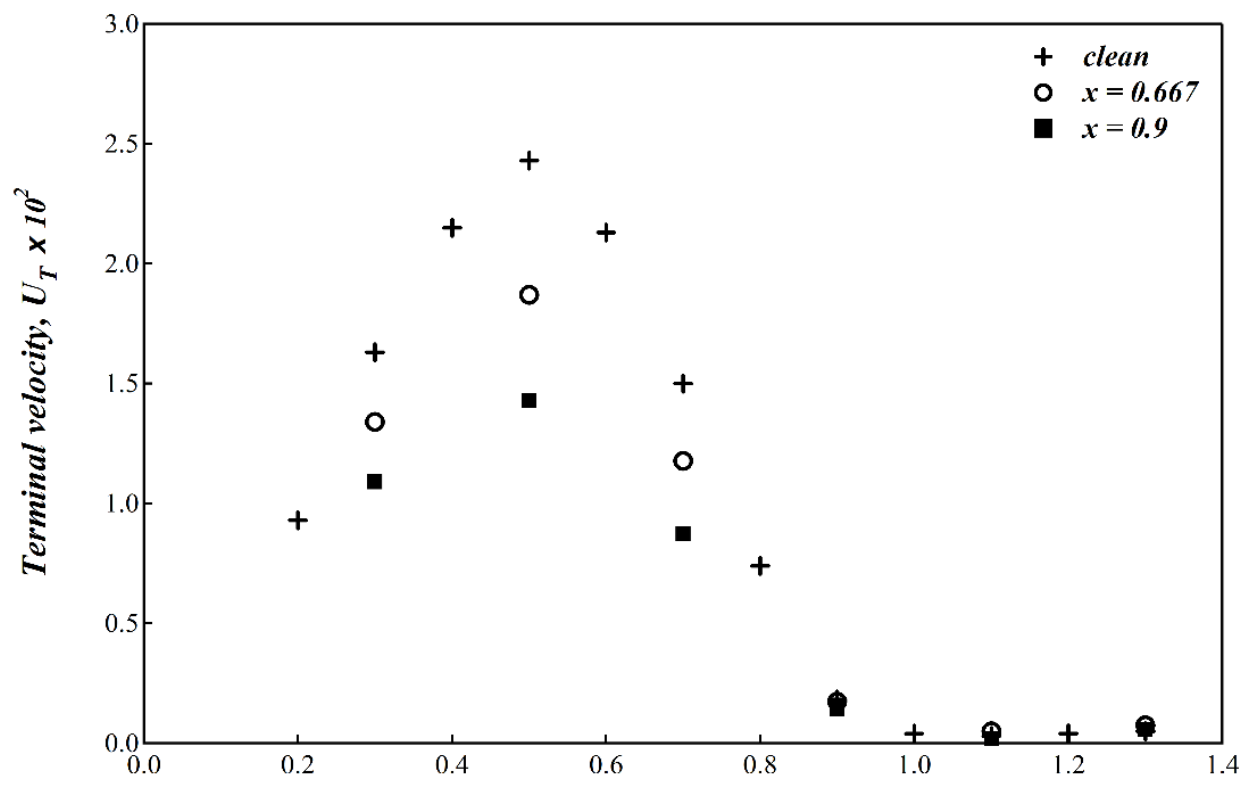

Drop size, $\kappa$

Figure 11: Comparison of terminal velocity as a function of drop size for $\mathrm{x}=0,0.667$, and 0.9 for $\chi=\lambda=0.1, R e=10, B o=1, B i=0.1$, and $P e_{s}=10$. 


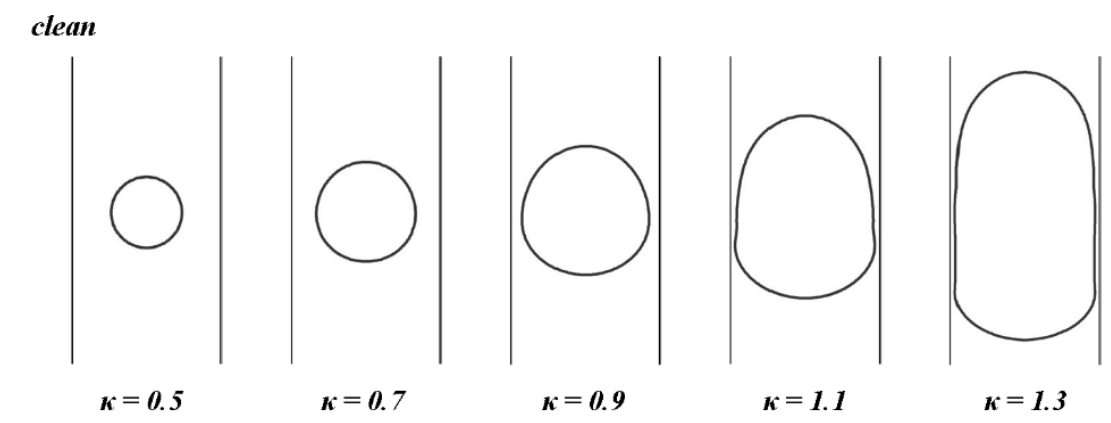

$x=0.9$

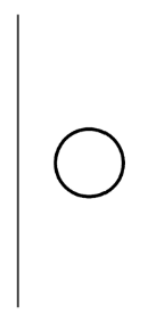

$\boldsymbol{\kappa}=0.5$
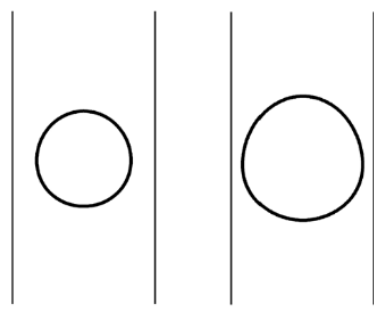

$\boldsymbol{\kappa}=\boldsymbol{0 . 7}$

$\boldsymbol{\kappa}=0.9$
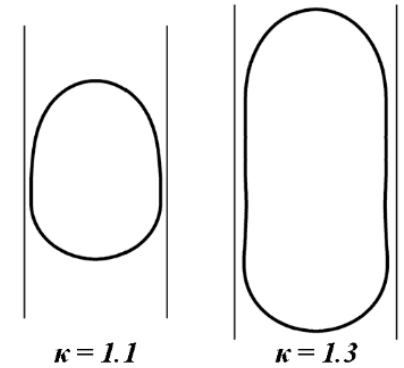

Figure 12: Comparison of steady drop shapes as a function of drop size for clean and $x=0.9$ drops with $\chi=\lambda=0.1, R e=10, B o=1, B i=0.1$, and $P e_{s}=10$.
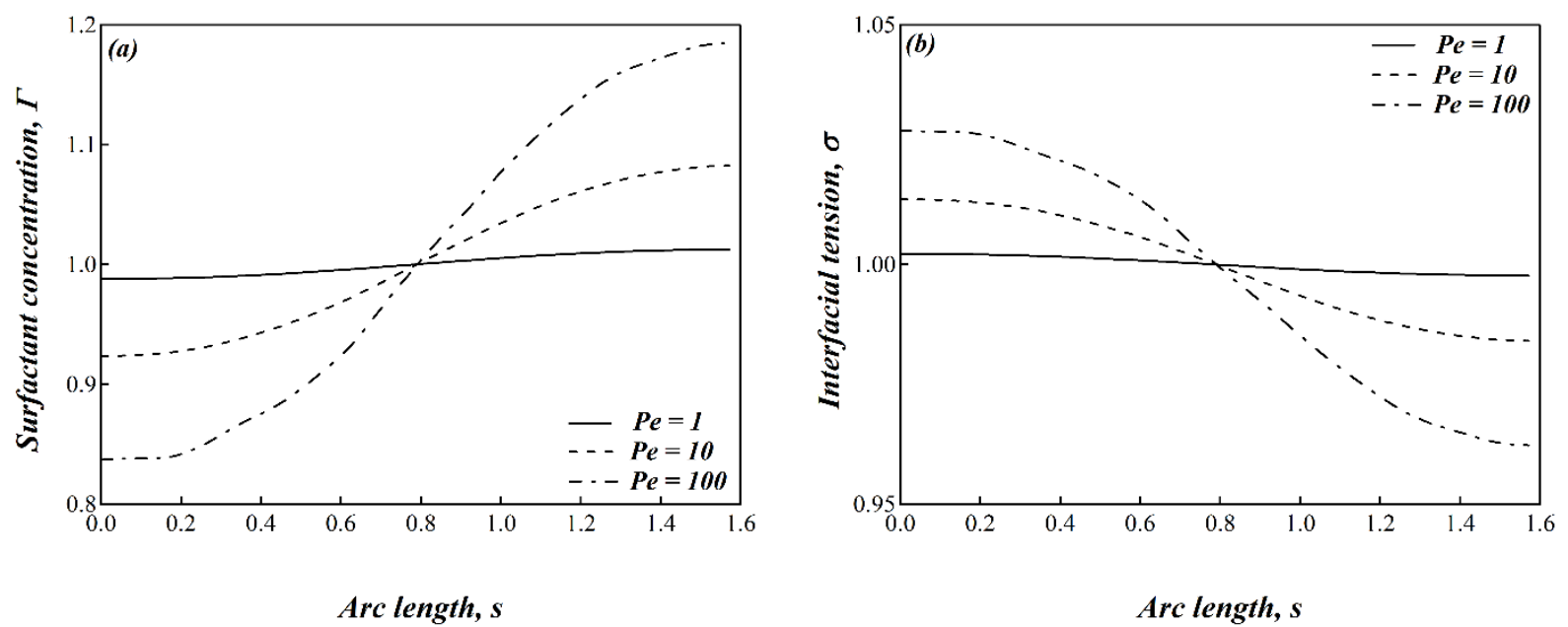

Figure 13: Effect of surface Peclet number on the (a) surfactant concentration and (b) interfacial tension along the interface at steady state for $\kappa=0.7, \chi=\lambda=0.1, R e=10, B o=1$, and $B i=0.1$.

\subsubsection{Effect of Peclet Number}

We end by considering the effect of the surface Peclet number on the buoyancy-driven motion of drops in the presence of surfactants. Figure 13 shows the surfactant concentration and the interfacial tension as a function of arc length for $P e_{s}=1,10$, and 100 for $\kappa=0.7, \chi=\lambda=0.1$, 
$R e=10, \quad B o=1$, and $B i=0.1$. Since the surface Peclet number represents the relative importance of the convective to diffusive transport of surfactant on the drop interface, interfacial diffusion dominates over convection at small $P e_{s}$ resulting in a nearly uniform interfacial tension along the drop interface as shown in Figure 13. As $P e_{s}$ increases, the interfacial tension gradient as well as the Marangoni stresses increases retarding the drop motion. The terminal velocity of drops as a function of $P e_{s}$ for $\kappa=0.5$ and 0.7 with $\chi=\lambda=0.1, R e=10, B o=1$, and $x=0.5$ and 0.9 is shown in Figure 14. Above a critical $P e_{s}(\sim 1000)$, the terminal velocity of the drop is independent of the surface Peclet number.

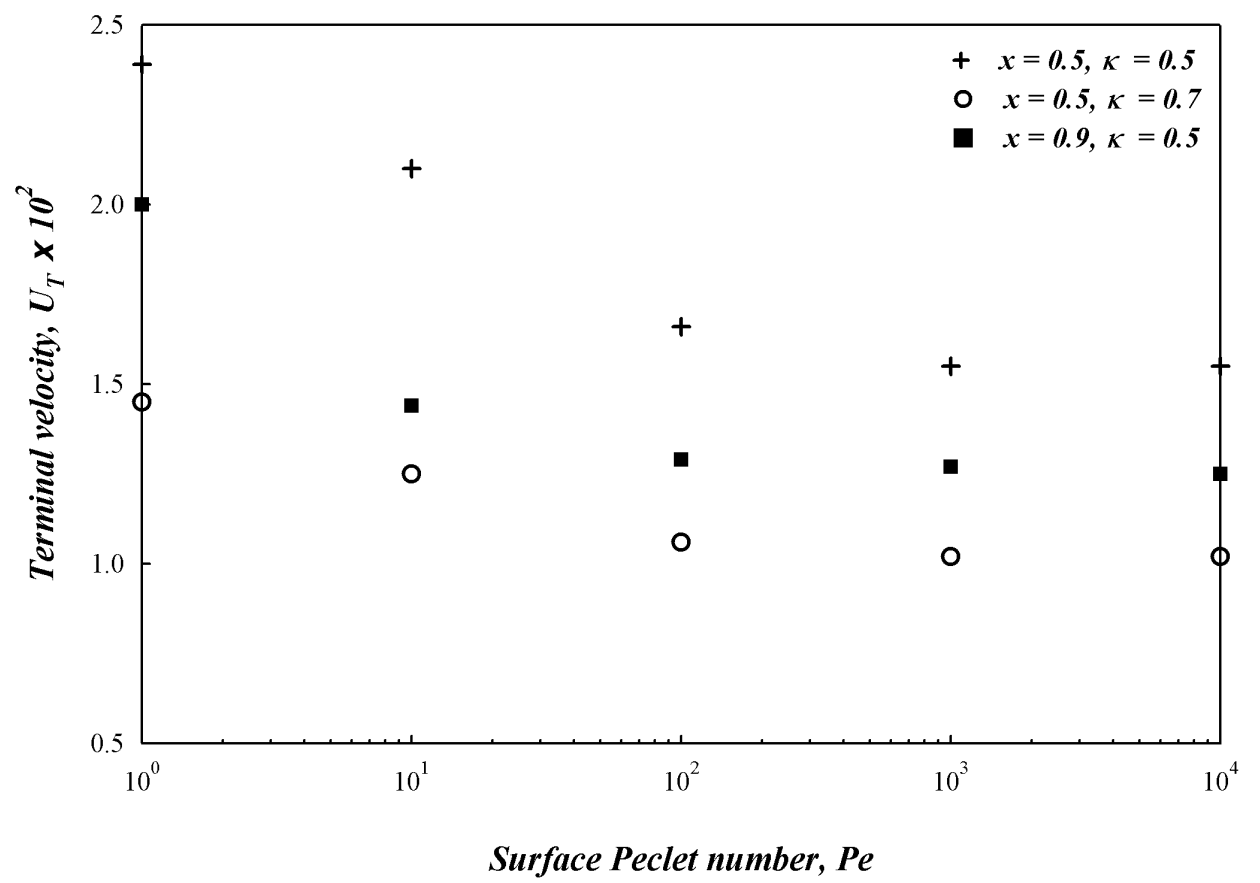

Figure 14: Terminal velocity of drops as a function of surface Peclet number with $\chi=\lambda=0.1$, $R e=10, B o=1$, and $B i=0.1$.

\section{Conclusions}

We have numerically studied the buoyancy-driven motion of drops rising through another quiescent immiscible fluid in a tube in the presence of soluble and insoluble surfactants. For the surfactant-free systems, we investigate the effects of changing Bond number and Weber number on the drop motion and shape at steady state for drops of different sizes. The terminal velocity of the drops increases with drop size first when drop size is small compared to the tube size. As the drop size becomes comparable to the capillary size, the increased wall drag reduces the velocity of the drops. Beyond a critical drop size, the drop terminal velocity is independent of the drop size. When the interfacial tension of the interface reduces by increasing the Bond number, the drop becomes more deformable resulting in more prolate shapes that travel faster than lower Bond number drops. Increasing inertial effects by increasing the Weber number results in flattening of the rear of the drop with the development of a negative curvature beyond a 
critical Weber number. For surfactant systems, the effect of the fractional coverage of surfactants, surfactant mass transfer to and from the interface, and the interfacial Peclet number on the velocity-volume curve was determined. The simulation results show that non-uniform distribution of surfactants along the interface gives rise to Marangoni stresses that oppose the interfacial flow and retards the drop motion. Larger Marangoni stresses generated when adsorption/desorption was limited or initial fractional coverage of surfactants was high, or interfacial Peclet number was large led to larger retardation of the drop velocity.

\section{Acknowledgements}

This work was supported by NSF grant CBET-0625425.

\section{References}

[1] R. Clift, J. R. Grace and M. E. Weber, Bubbles, Drops, and Particles, Academic Press, 1978.

[2] F. Bretherton, "The motion of long bubbles in tubes.," Journal of Fluid Mechanics, vol. 10, pp. 166-188, 1961.

[3] H. L. Goldsmith and S. G. Mason, "The movement of single large bubbles in closed vertical tubes," Journal of Fluid Mechanics, vol. 14, pp. 42-58, 1962.

[4] E. T. White and R. H. Beardmore, "The velocity of rise of single cylindrical air bubbles through liquids contained in vertical tubes," Chemical Engineering Science, vol. 17, pp. 351-361, 1962.

[5] M. Coutanceau and P. Thizon, "Wall effects on the bubble behavior in highly viscous liquids," Journal of Fluid Mechanics, vol. 107, pp. 339-373, 1981.

[6] M. Coutanceau and A. Texier, "Experimental investigation of the creeping motion of a drop in a vertical tube," Experiments in Fluids, vol. 4, pp. 241-246, 1986.

[7] D. A. Reinelt, "The rate at which a long bubble rises in a vertical tube.," Joutrnal of Fluid Mechanics, vol. 175, pp. 557-565, 1987.

[8] A. Borhan and J. Pallinti, "Buoyancy-driven motion of viscous drops through cylindrical capillaries at small Reynolds numbers.," Industrial \& Engineering Chemistry Research, vol. 34, pp. 2750-2761, 1995.

[9] F. Viana, R. Pardo, R. Yanez, J. L. Trallero and D. D. Joseph, "Universal correlation for the rise velocity of long gas bubbles in round pipes.," Journal of Fluid Mechanics, vol. 494, pp. 379-398, 2003.

[10] J. Li, V. Bulusu and N. R. Gupta, "Buoyancy-driven motion of bubbles in square channels," Chemical Engineering Science, vol. 63, pp. 3766 - 3774, 2008. 
[11] L. Bozzi, J. Feng, T. Scott and A. Pearlstein, "Steady axisymmetric motion of deformable drops falling or rising through a homoviscous fluid in a tube at intermediate Reynolds number.," Journal of Fluid Mechanics, vol. 336, pp. 1-32, 1997.

[12] T. Taha and Z. Cui, "CFD modelling of slug flow in vertical tubes.," Chemical Engineering Science, vol. 61, pp. 676-687, 2006.

[13] J. Feng, "Buoyancy-driven motion of a gas bubble through viscous liquid in a round tube," Journal of Fluid Mechanics, vol. 609, pp. 377-410, 2008.

[14] K. Hayashi and A. Tomiyama, "A drag correlation of fluid particles rising through stagnant liquids in vertical pipes at intermediate Reynolds numbers," Chemical Engineering Science, vol. 64, pp. 3019-3028, 2009.

[15] E. Almatroushi and A. Borhan, "Surfactant effect on the buoyancy-driven motion of bubbles and drops in a tube," Annals of the New York Academy of Sciences, vol. 1027, pp. 330-341, 2004.

[16] P. Daripa and G. Pasa, "The effect of surfactant on long bubbles rising in vertical capillary tubes," Journal of Statistical Mechanics: Theory and Experiment, p. L02003, 2011.

[17] S. Tasoglu, U. Demirci and M. Muradoglu, "The effect of soluble surfactant on the transient motion of a buoyancy-driven bubble," Physics of Fluids, vol. 20, p. 040805, 2008.

[18] K. Hayashi and A. Tomiyama, "Effects of surfactant on terminal velocity of a Taylor bubble in a vertical pipe," International Journal of Multiphase Flow, vol. 39, pp. 78-87, 2012.

[19] R. Kurimoto, K. Hayashi and A. Tomiyama, "Terminal velocities of clean and fullycontaminated drops in vertical pipes," International Journal of Multiphase Flow, vol. 49, pp. 8-23, 2013.

[20] F. Jin, N. R. Gupta and K. J. Stebe, "The detachment of a viscous drop in a viscous solution in the presence of a soluble surfactant," Physics of Fluids, vol. 18, p. 0222103, 2006.

[21] H. Stone, "A simple derivation of the time-dependent convective-diffusion equation for surfactant transport along a deforming interface," Physics of Fluids A, vol. 2, pp. 111-112, 1990.

[22] H. R. Wong and C. Maldarelli, "On the surfactant mass balance at a deforming fluid interface," Physics of Fluids, vol. 8, pp. 3203-3204, 1996.

[23] S. Popinet and S. Zaleski, "A front-tracking algorithm for accurate representation of surface tension," International Journal for Numerical Methods in Fluids, vol. 30, pp. 775-793, 1999.

[24] F. Jin and K. J. Stebe, "The effects of a diffusion-controlled surfactant on a viscous drop 
injected into a viscous medium," Physics of Fluids, vol. 19, p. 112103, 2007.

[25] M. Muradoglu and G. Tryggvason, "A front-tracking method for computation of interfacial flows with soluble surfactants," Journal of Computational Physics, vol. 227, pp. 2238-, 2008.

[26] Y. Cui and N. R. Gupta, "Drop formation in co-flowing fluid streams.," International Journal of Transport Phenomena, vol. 12, pp. 217-226, 2011.

[27] Y. Cui and N. R. Gupta, "Surfactant effects on drop formation in co-flowing fluid streams.," Colloids and Surfaces A, vol. 393, pp. 111-121, 2012.

[28] D. Gueyffier, J. Li, A. Nadim, R. Scardovelli and S. Zaleski, "Volume-of-fluid interface tracking with smoothed surface stress methods for three-dimensional flows," Journal of Computational Physics, vol. 152, pp. 423-456, 1999.

[29] J. U. Brackbill, D. B. Kothe and C. Zemach, "A continuum method for modeling surface tension," Journal of Computational Physics, vol. 100, pp. 335-, 1992.

[30] G. Ryskin and L. Leal, "Numerical solution of free-boundary problems in fluid mechanics. Part 2. Buoyancy-driven motion of a gas bubble through a quiescent liquid," Journal of Fluid Mechanics, vol. 148, pp. 19-35, 1984.

[31] C. D. Eggleton and K. J. Stebe, "An adsortion-desorption-controlled surfactant on a deforming droplet," Journal of Colloid and Interface Science, vol. 208, pp. 68-80, 1998. 